\title{
Crecimiento, supervivencia y herbivoría de plántulas de Brosimum alicastrum (Moraceae), una especie del sotobosque neotropical
}

\author{
H.S. Ballina-Gómez ${ }^{1}$, S. Iriarte-Vivar ${ }^{1,2}$, R. Orellana ${ }^{1} \&$ L.S. Santiago ${ }^{3}$ \\ 1. Unidad de Recursos Naturales Centro de Investigación Científica de Yucatán A.C. Calle 43 No. 130 Chuburna de \\ Hidalgo 97200, Mérida, Yucatán, México; hballina@cicy.mx \\ 2. Dirección actual: Facultad de Ciencias, Departamento de Ecología y Recursos Naturales, Universidad Nacional \\ Autónoma de México, Circuito Exterior, Ciudad Universitaria, C.P. 04510, México, D.F. \\ 3. Department of Botany and Plant Sciences, University of California, Riverside, CA92521, EEUU; mairivi@yahoo. \\ com.mx; orellana@cicy.mx; santiago@ucr.edu
}

Recibido 24-I-2008. Corregido 30-VI-2008. Aceptado 31-VII-2008.

\begin{abstract}
Growth, survival and herbivory of seedlings in Brosimum alicastrum (Moraceae), a species from the Neotropical undergrowth. Growth responses, survival, and herbivory, on seedlings of Brosimum alicastrum were studied in a neotropical Mexican forest. We selected 122 seedlings and divided them into three groups assigned to defoliation treatments: control or $0(\mathrm{n}=21), 50(\mathrm{n}=51)$ and $90 \%(\mathrm{n}=50)$. Every 4 months during two years we measured seedling growth (in terms of relative growth rate in biomass, leaf area growth, produced leaves and height growth) and survival. In addition, we evaluated every 12 months pathogen damage and insect herbivory using a $2 \mathrm{~mm}^{-2}$ grid. Separately, we estimated mammal herbivory in 3-month old seedlings that were selected within a plot of $500 \mathrm{~m} \times 10 \mathrm{~m}(\mathrm{~N}=1095)$. Pathogen damage and insect herbivory were evaluated within the same plot in 113 seedlings. We found that $50 \%$ defoliated seedlings showed compensatory responses in all growth parameters. Relative growth rate and height growth also had a compensatory response in seedlings at $90 \%$ defoliation. Relative growth rate and leaf area growth gradually decreased with time although height growth seedling showed an opposite pattern. Leaves produced were not affected by time. Estimated seedling survival probability increased with defoliation to a maximum of $97 \%$, decreasing at 24 month to $37 \%$. Mammal herbivory was more frequent and severe than herbivory caused by pathogens and insects. In some cases, mammal herbivory produced total defoliation. Compensatory growth in leaf area growth, produced leaves and height growth seedling suggest a synergic compensatory mechanism expressed in a whole-plant growth biomass (relative growth rate). Compensation and survival results suggest trade-offs at the leaf level, such as leaf area growth and produced leaves versus chemical defenses, respectively. Rev. Biol. Trop. 56 (4): 2055-2067. Epub 2008 December 12.
\end{abstract}

Keywords: Brosimum alicastrum, defoliation treatments, growth compensatory, herbivory, relative growth rate, seedling survival.

En las selvas tropicales las plantas se enfrentan a pérdidas de biomasa causadas por herbívoros insectos (Huntly 1991), patógenos (Augspurger 1984) y mamíferos (Augspurger y Kitajima 1992). Esta pérdida de biomasa puede tener un gran impacto sobre el crecimiento y la reproducción de las plantas (Marquis 1984, Crawley 1989). Se ha estimado que en las selvas tropicales aproximadamente el $11 \%$ del área foliar anual es consumida por herbívoros y patógenos (Coley y Aide 1991). Generalmente los estados más vulnerables a la herbivoría pueden situarse en dos categorías: hojas jóvenes durante el ciclo de vida de una hoja, y plántulas durante el ciclo de vida de una planta. En especies tropicales tolerantes a la sombra se ha visto que sus hojas nuevas en expansión pueden sufrir de 5 a 100 veces más daño por parte de patógenos y herbívoros, que sus hojas maduras (Coley y Aide 1991). 
El modelo de Janzen (1970) y Connell (1971) postula que las plántulas encontradas más próximas al árbol progenitor presentarán mayores tasas de mortalidad que aquellas más lejanas. Esta mortalidad es resultado de los patógenos e insectos herbívoros asociados al árbol progenitor sin un daño aparente. La presión de estos organismos puede aumentar de manera denso-dependiente, disminuyendo el éxito en el reclutamiento de las plántulas e influir en la diversidad de especies vegetales (Howe y Smallwood 1982). Sin embargo, conforme las plántulas van creciendo e incrementando su edad y tamaño, la mortalidad disminuye (Howe 1990, Augspurger y Kitajima 1992).

Los efectos de la herbivoría muchas veces se han reportado como negativos (Stone y Bacon 1995) debido principalmente a la reducción del área foliar fotosintéticamente activa que sufren las plantas. Aunque la caída de ramas del dosel también puede contribuir a perder importantes cantidades de área foliar (Clark y Clark 1989). Coley et al. (1985) en su hipótesis de la disponibilidad de recursos mencionan que después de un evento de herbivoría las especies tolerantes a la sombra asignarían sus recursos hacia defensa en detrimento de su crecimiento. Como resultado, el crecimiento resultaría afectado negativamente de manera que el crecimiento sería menor en comparación a plantas sin herbivoría.

Algunos resultados opuestos mencionan que una planta después de un evento de herbivoría podría incrementar a corto plazo el crecimiento de su parte aérea (McNaughton 1983, Hjalten et al. 1994). Incluso este incremento sería hasta el punto de igualar (compensar) o rebasar (sobrecompensar) el crecimiento de una planta que no sufrió tal herbivoría (McNaughton 1983). El crecimiento compensatorio ya ha sido reportado en diversas especies (Parra-Tabla et al. 2004, Massey et al. 2005), no obstante aún no se han encontrado reportes en relación al crecimiento individual expresado en la tasa relativa de crecimiento de las plantas (Relative Growth Rate, $\mathrm{RGR}_{\text {Biomasa }}$ ), medida más usada de desempeño en plantas debido a su alta sensibilidad al daño causado por patógenos y herbívoros (Poorter 1989), ya que es el resultado de varios procesos metabólicos de las estructuras vegetativas de la planta (Poorter 2002).

En este trabajo se estudió en condiciones naturales el efecto de la defoliación artificial sobre el crecimiento total de Brosimum alicastrum C.C. Berg (1972), supervivencia y sus principales herbívoros, dentro de una selva subhúmeda tropical en Calakmul, Campeche, México. B. alicastrum por ser una especie tolerante a la sombra se esperaría que sus plántulas con defoliación artificial asignen los recursos disponibles a otras funciones más que a su crecimiento, lo que se expresaría en un menor crecimiento y supervivencia, patrón opuesto en las plántulas no defoliadas.

Se plantearon cuatro objetivos: 1. Analizar en condiciones de sotobosque, el desempeño de las plántulas en función de su crecimiento total y de otras estructuras vegetativas como el área foliar, hojas producidas y altura, bajo el efecto de una defoliación artificial, 2. Determinar si la supervivencia de las plántulas es afectada por el grado de severidad de una defoliación artificial, 3. Determinar si las respuestas de crecimiento y supervivencia de las plántulas con la defoliación artificial son influenciadas por el factor tiempo, 4. Evaluar los tipos de herbivoría que presentan las plántulas de esta especie y cuantificar sus grados de severidad en una selva subhúmeda tropical de Calakmul, Campeche, México.

\section{MATERIALES Y MÉTODOS}

Sitio de estudio y especie: El trabajo se realizó en una selva subhúmeda perteneciente a la Reserva de la Biosfera de Calakmul $\left(18^{\circ}\right.$ $18^{\prime} 47^{\prime \prime} \mathrm{N}$ y $89^{\circ} 51^{\prime} 48^{\prime \prime} \mathrm{O}, 240 \mathrm{~m}$ altitud) localizada al sureste del estado de Campeche en el municipio de Calakmul. La precipitación y temperatura media anual son de $1141 \mathrm{~mm}$ y $25^{\circ} \mathrm{C}$, respectivamente (García 1988). Este tipo de selva contiene comunidades vegetales de 15 a 25 m altura las cuales de un 25 a $50 \%$ de las especies pierden sus hojas (Arriaga et al. 2000, Morales y Magaña 2001). La riqueza 
de especies de plantas estimada es de 1569 a 1600 (Carabias et al. 2000, Martínez et al. 2001). Esta región se caracteriza por tener una marcada estacionalidad dividida en tres temporadas: nortes (octubre-enero), secas (febreromayo) y lluvias (junio-septiembre/noviembre) (Martínez y Galindo-Leal 2002, Orellana et al. 2003). Los suelos que predominan son del tipo cárstico, con abundantes afloramientos de calcio y menos ácidos que otras selvas tropicales (Wendt 1993).

B. alicastrum es una especie perennifolia de sotobosque, de lento crecimiento, y de larga vida, que alcanza una altura hasta de $45 \mathrm{~m}$ y un diámetro a la altura del pecho (DAP) de hasta $1.5 \mathrm{~m}$. Se distribuye desde el sur de México hasta Colombia, Perú y Venezuela y en las Islas del Caribe. En la selva subhúmeda de Calakmul se han encontrado grandes bancos de plántulas (H. Ballina-Gómez, Obs. personal). Reportes en la literatura mencionan daños por patógenos y algunos tipos de insectos, sin llegar a ser evidente un efecto como la disminución de su crecimiento (De la Cruz y Dirzo 1987).

Tratamientos de defoliación: A finales de Abril y principios de Mayo de 2005 se seleccionaron cuatro sitios dentro de una extensa población de $B$. alicastrum. Los criterios de selección de estos sitios fueron: áreas de selva con baja perturbación e igual porcentaje de luz sobre el sotobosque (datos no mostrados). Se marcaron 122 plántulas de $B$. alicastrum, únicamente se seleccionaron plántulas con alturas de entre 15 y $30 \mathrm{~cm}$, número de hojas entre 3 y 10 , sin síntomas considerables de enfermedad (determinado visualmente por el vigor de las plántulas y el color verde de sus hojas). Se asignaron tres tratamientos de defoliación de manera aleatoria: 1 . control ( $0 \%$ defoliación) $(n=21)$, en el cual no se removió ninguna hoja sobre la plántula; $2.50 \%$ defoliación $(\mathrm{n}=51)$, en este se removieron las hojas de manera intercalada, iniciando con la remoción de la segunda hoja más joven totalmente expandida; y 3. $90 \%$ defoliación $(n=50)$, se removieron casi todas las hojas, excepto la más nueva. La remoción se realizó a partir de la base de la lámina foliar dejando intacto el pecíolo. Sólo se seleccionaron 21 plántulas en el tratamiento control debido a la falta de plántulas que se ajustaran a los criterios antes descritos.

A todas las hojas removidas se les midió su área foliar para determinar los porcentajes de defoliación aplicados. Las hojas removidas se conservaron húmedas en el laboratorio y se midió el largo, ancho y área de cada hoja con un medidor de área foliar (Modelo Li-3100; Li-Cor, Inc., Lincoln, NE, USA, 1996).

Estimación del área foliar y la biomasa: $\mathrm{Al}$ inicio del estudio se recolectaron siete plántulas registrando sus alturas, diámetros basales, número de hojas y área foliar de cada hoja, utilizando el medidor de área foliar. También se obtuvo la biomasa seca de las hojas, tallos y raíces utilizando un secador a $55^{\circ} \mathrm{C}$ hasta alcanzar un peso constante. De una muestra total de 500 hojas se estimó un modelo de regresión lineal simple para el área foliar (AF) de cada hoja con respecto al largo de las hojas $(\mathrm{LH})$ obteniéndose la siguiente ecuación $\mathrm{AF}=$ $-8.2501+2.6362 * \mathrm{LH}\left(\mathrm{R}^{2}=0.97, \mathrm{P}<0.0001\right)$ con la cual se calculó el área foliar en los subsecuentes periodos de medición.

De un total de 67 plántulas, se estimaron modelos de regresiones lineales para la biomasa total de cada plántula con respecto a la altura $\left(\mathrm{R}^{2}=0.4\right)$, área foliar $\left(\mathrm{R}^{2}=0.84\right)$, diámetro $\left(\mathrm{R}^{2}=\right.$ $0.79)$ y número de hojas $\left(\mathrm{R}^{2}=0.99\right)$. La variable con mejor desempeño en la estimación de la biomasa total (BT) fue el número de hojas por plántula $(\mathrm{H})$, con la siguiente ecuación $\mathrm{BT}=$ $\left.0.6446 * \mathrm{H}-1.2293, \mathrm{R}^{2}=0.99, \mathrm{P}<0.0001\right) . \mathrm{De}$ esta manera el número de hojas por plántula se seleccionó como el parámetro para estimar la biomasa total en cada periodo de medición.

Análisis de crecimiento: Cada cuatro meses, comenzando a principios de Mayo de 2005 se obtuvieron las siguientes medidas: altura (desde el diámetro basal hasta el meristemo apical), número de hojas nuevas, y largo de hojas.

Además, a partir de la biomasa estimada se calculó la $\mathrm{RGR}_{\text {Biomasa }}$ promedio para cada plántula $\left(\mathrm{g} \mathrm{g}^{-1}\right.$ día $\left.^{-1}\right)$ según Hunt (1978): 


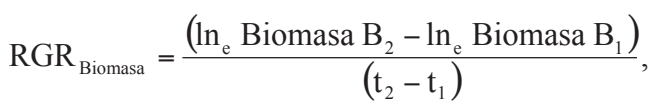

donde: $\mathrm{B}_{1}=$ biomasa inicial, $\mathrm{B}_{2}=$ biomasa final, $t_{1}=$ tiempo cero o inicio del experimento, $t_{2}=$ días transcurridos al tiempo de cosecha.

Estimación de herbivoría: Se estimó la herbivoría en dos poblaciones: (1) plántulas experimentales (PE) (plántulas sometidas a los tres tratamientos de defoliación artificial y (2) plántulas no experimentales (PNE) (plántulas que no intervinieron en los tratamientos de defoliación). En PE, se evaluó la herbivoría por patógenos e insectos cada 12 meses durante dos años. En cada censo se registró el área foliar perdida de cada hoja con una cuadrícula de $2 \mathrm{~mm}^{-2}$. En PNE, se estimó la herbivoría por mamíferos, insectos y patógenos en diciembre de 2006 sobre plántulas provenientes de semillas marcadas durante el periodo de fructificación en septiembre de 2006 dentro de una parcela de $500 \mathrm{~m}$ de largo x $10 \mathrm{~m}$ de ancho, con similares condiciones de luz y grado de perturbación que los cuatro sitios con las PE. Este proceso permitió conocer la edad aproximada de las plántulas ( 3 meses) y eliminar la sobre estimación de la herbivoría por efecto acumulativo en el tiempo (Coley y Barone 1996). Las plántulas para evaluar la herbivoría por mamíferos se ubicaron dentro de 50 cuadros de $1 \mathrm{~m}^{2}$ trazados de manera aleatoria. Se consideró a una plántula con esta herbivoría (ramoneo) cuando presentó remoción de la yema apical y hojas. Las plántulas para evaluar la herbivoría por patógenos e insectos fueron 113 y las condiciones de selección fueron alturas de 15 y 30 $\mathrm{cm}$, cuando menos tres hojas y un buen estado vigoroso (determinado visualmente por el color verde de las hojas). Se recolectaron las dos primeras hojas más cercanas al suelo (total $\mathrm{n}=$ 226) y con el medidor de área foliar a cada hoja se le midió el área presente y el área faltante o con necrosis la cual se estimó dibujándose sobre un papel blanco. El área foliar total por hoja fue la suma del área foliar presente más el área faltante. Se identificó la herbivoría por patógenos (bacterias y virus) como el área foliar con huellas de necrosis. Se consideró a una plántula con herbivoría por insectos cuando presentó en sus hojas, remoción de área foliar característica de insectos defoliadores, raspadores, agalladores y minadores.

Análisis estadístico de datos: Los datos de crecimiento y supervivencia se analizaron utilizando modelos lineares generalizados (MLG, McCullagh y Nelder 1989). El crecimiento en las cuatro variables de respuesta fue analizado bajo una estructura de medidas repetidas con un factor (tratamiento de defoliación, con tres niveles) y un factor intra-sujetos (tiempo en meses, con 4 niveles). La RGR $_{\text {Biomasa }}$ con un modelo probabilístico Normal con una función de enlace Identidad. El área foliar y altura con modelos probabilísticos Gamma con funciones de enlace Log; las hojas producidas con uno de Poisson y una función de enlace Log. Las diferencias entre los tres niveles del factor defoliación (control, 50 y 90\%) y entre los cuatro niveles intra-sujetos (tiempo a 4, 8, 12 y 24 meses) se analizaron usando comparaciones pareadas de Bonferroni.

La probabilidad de supervivencia se estimó en las plántulas defoliadas a los 4, 8, 12 y 24 meses de iniciado el experimento. En el caso de los tratamientos de defoliación se usó al tratamiento control como nivel de referencia y para el tiempo la supervivencia a los 4 meses. Se usó el modelo probabilístico Binomial (viva/muerta) con una transformación de la variable a logit. Se aplicó entonces un modelo de regresión logística considerando a la supervivencia como variable de respuesta, al tiempo y a la defoliación como factores de covariación. Se estimaron los coeficientes del modelo. Se usó el estadístico de chi $^{2}$ de Wald, bajo el método de máxima verosimilitud, para probar el efecto de los factores de covariación, defoliación y tiempo, sobre la supervivencia de las plántulas. La bondad de ajuste del modelo se realizó analizando los residuos de la desviación de los valores de supervivencia. Todos los análisis estadísticos se realizaron con el paquete estadístico SPSS 
16 para Windows (SPSS 2007) y Statistica 7.0 (StatSoft 2004) para Windows.

\section{RESULTADOS}

Crecimiento: En la $\mathrm{RGR}_{\text {Biomasa }}$ la interacción de los factores defoliación $\times$ tiempo fue significativa. De manera independiente sólo el factor tiempo presentó efectos significativos (Cuadro 1). A los 4 meses las plántulas sometidas a todos los tratamientos obtuvieron las mayores $\mathrm{RGR}_{\text {Biomasa' }}$ pero entre el resto de meses estas fueron similares (Fig. 1A). Entre tratamientos únicamente a los 4 meses las RGR $_{\text {Biomasa }}$ fueron mayores en el control con respecto a los dos tratamientos de defoliación (Bonferroni $\mathrm{P}<0.05-0.005$ ) (Fig. 1A). En el área foliar la interacción defoliación $\times$ tiempo no fue significativa, pero ambos factores de manera independiente sí (Cuadro 1). El crecimiento del área foliar fue similar entre el control y $50 \%$ de defoliación, pero en ambos casos mayor al obtenido con el 90\% de defoliación (Cuadro 1). El área foliar disminuyó gradualmente con el tiempo teniendo su menor crecimiento a los 24 meses en comparación con los meses anteriores (Bonferroni $\mathrm{P}<0.05-0.01$ ) (Fig. 1B). En el número de hojas producidas la interacción defoliación $\times$ tiempo fue marginalmente significativa, en cambio sólo la defoliación la afectó significativamente (Cuadro 1). El número de hojas producidas fue 2 veces más en el control y casi una vez mayor en el 50\% de defoliación, que con el 90\% de defoliación (Bonferroni $\mathrm{P}<0.005$ ) (Cuadro 1). A los 4, 8 y 12 meses el número de hojas disminuyó significativamente con la defoliación (Bonferroni $<0.05-0.0001$ ). Aunque a los 24 meses no hubo diferencias significativas entre el control y los tratamientos de defoliación (Fig. 1C). En la altura, ni la interacción defoliación $\times$ tiempo ni el factor defoliación influenciaron significativamente la altura, únicamente lo hizo el tiempo (Cuadro 1). Las plántulas obtuvieron alturas mayores a los 12 meses, y las menores a los 4 y 8 meses (Bonferroni $<0.05-0.0001$ ) (Fig. 1D).

Supervivencia de plántulas: La supervivencia de las plántulas excedió el $60 \%$ en todos

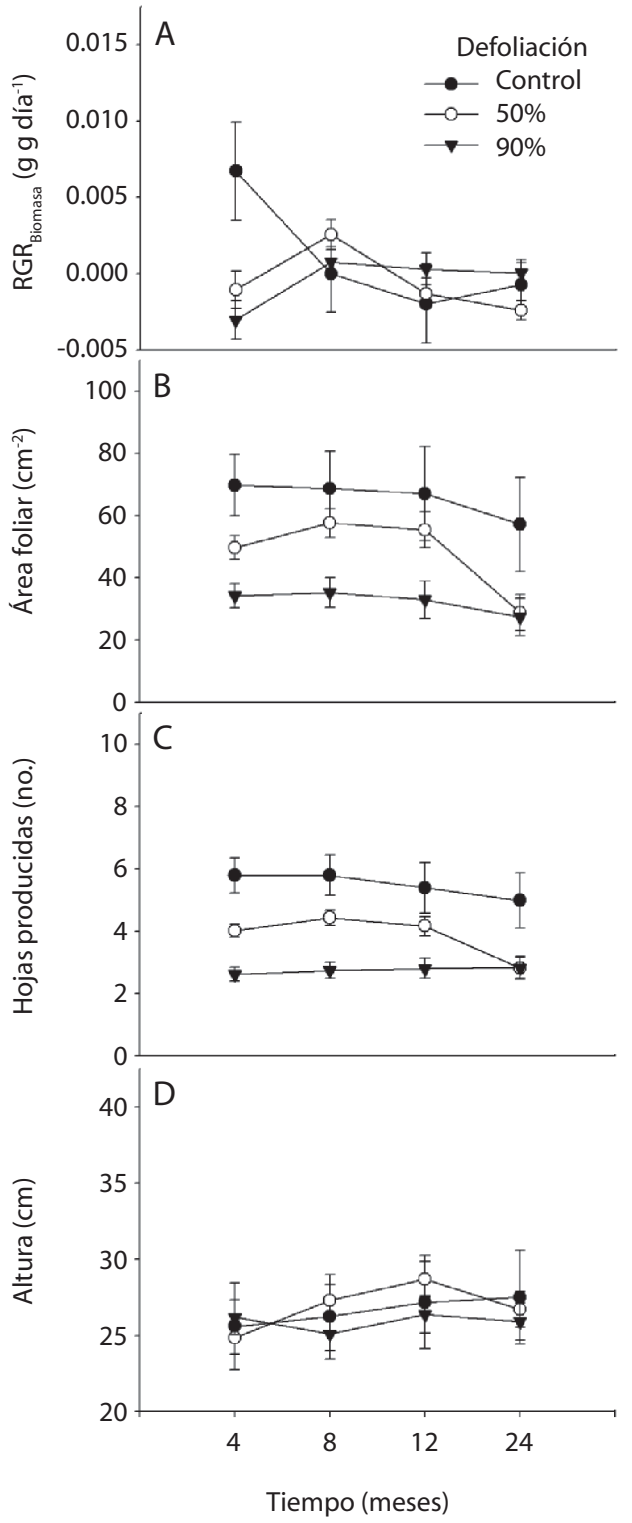

Fig. 1. Crecimiento en $\mathrm{RGR}_{\text {Biomasa }}(\mathrm{A})$, área foliar (B), producción de hojas (C) y altura (D) en plántulas de $B$. alicastrum sometidas a defoliación.

Fig. 1. Seedling growth of B. alicastrum in terms of $\mathrm{RGR}_{\text {Biomass }}(\mathrm{A})$, leaf area (B), leaf production (C) and height (D) affected by defoliation. 
los tratamientos durante los dos años de estudio. Las plántulas sometidas a los tratamientos de defoliación y control difirieron significativamente en su supervivencia de manera que en las plántulas defoliadas fue mayor (Cuadro 2, Fig. 2). El tiempo también afectó significativamente la supervivencia yendo desde un $97-60 \%$ a los 4 meses hasta disminuir a un $78-37 \%$ a los 24 (Cuadro 2, Fig. 2).

Herbivoría en plántulas de $\boldsymbol{B}$. alicastrum: En PE, durante el primer año de estudio de 471 hojas analizadas el 40\% sufrió algún tipo de herbivoría por insectos o patógenos. Sin embargo, el área foliar removida de todas las hojas fue de apenas $4.4 \%$. El porcentaje promedio de herbivoría en las hojas fue $12.15 \%$. Los porcentajes de herbivoría presentaron las siguientes frecuencias porcentuales: $70 \%$ en hojas sin herbivoría hasta con un $10 \%$ y menos del $20 \%$ en hojas con herbivoría de 10.1 a $100 \%$. En el segundo año de estudio de 268 hojas analizadas sólo el 10\% sufrió algún tipo de herbivoría por insectos o patógenos. El área total removida fue de sólo $8.4 \%$. El porcentaje promedio de herbivoría en las hojas fue de $47.19 \%$. Los porcentajes de herbivoría presentaron las siguientes frecuencias (\%): $33 \%$ en hojas sin herbivoría hasta con un $10 \%, 38 \%$ en hojas con herbivoría de 50.1 a $100 \%$ y con menos del $20 \%$ en hojas con herbivoría de 10.1 a $50 \%$. Generalmente las hojas nuevas producidas a lo largo de los dos años de estudio no presentaron algún tipo de herbivoría. La mayoría de las hojas con herbivoría fueron aquellas presentes desde el inicio del estudio (Fig. 3).

En PNE de 1095 plántulas analizadas para la herbivoría por mamíferos, el 27.4\% (300 plántulas) presentó daños por ramoneo. Para la herbivoría por insectos y patógenos de 226 hojas analizadas, 146 presentaron al menos algún tipo de daño (65\%) y 9 hojas (4\%) presentaron dos tipos de daño. En este último caso en 8 hojas los daños fueron causados por insectos raspadores y defoliadores. El principal tipo de daño fue el causado por patógenos, presente en 125 hojas (55\%). La herbivoría por insectos defoliadores y raspadores se encontró en 16

\section{CUADRO 2}

Regresión logística analizada mediante un modelo lineal generalizado para estimar la supervivencia de las plántulas de B. alicastrum. Se incluye el tiempo y los tratamientos de defoliación como variables categóricas. Se muestran para cada variable sus estimados beta ( $\beta \pm$ Error estándar), nivel de significancia (análisis de Wald), valores Exp ( $\beta$ ), intervalos de confianza (95\%), los residuos de las desviaciones de la variable de respuesta y sus grados de libertad (G.L.)

TABLE 2

Results of a logistic regression analyzed through a GLM model to predict seedlings survival of B. alicastrum. We included time and defoliation treatments as categorical variables. In each variable we showed beta parameters estimates ( $\beta \pm$ standard error), significance level (Wald test), Exp ( $\beta$ ), confidence interval (95\%), deviance residual and its degrees of freedom (D.F.)

$\begin{array}{lcccccccccc}\begin{array}{l}\text { Factores y sus } \\ \text { niveles }\end{array} & \beta & \text { E.E. } & \text { Wald } & \text { G.L. } & \text { P } & \text { Exp }(\beta) & \begin{array}{c}\text { Intervalos de } \\ \text { confianza (95\%) }\end{array} & \begin{array}{c}\text { Residuos } \\ \text { de la } \\ \text { desviación }\end{array} & \text { G.L. } \\ \text { Tiempo } & & & & & & & & \text { Inferior } & \text { Superior } & \\ \text { Tiempo (8 meses) } & 1.448^{-16} & 0.454 & 1.016^{-31} & 1 & 1 & 1 & 0.410 & 2.437 & \\ \text { Tiempo (12 meses) } & -0.437 & 0.422 & 1.069 & 1 & 0.301 & 0.646 & 0.283 & 1.478 \\ \text { Tiempo (24 meses) } & -1.983 & 0.376 & 27.858 & 1 & \mathrm{P}<0.0001 & 0.138 & 0.066 & 0.287 \\ \text { Defoliación } & & & 28.327 & 2 & \mathrm{P}<0.0001 & & & & \\ \text { Defoliación (50\%) } & 1.690 & 0.333 & 25.756 & 1 & \mathrm{P}<0.0001 & 5.419 & 2.821 & 10.407 \\ \text { Defoliación (90\%) } & 1.356 & 0.320 & 17.934 & 1 & \mathrm{P}<0.0001 & 3.880 & 2.072 & 7.267\end{array}$




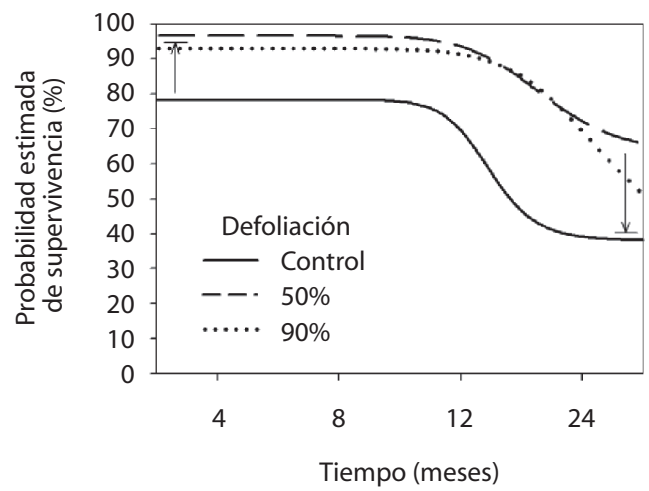

Fig. 2. Regresión logística analizada con un GLM para estimar la probabilidad de supervivencia como una función del porcentaje de defoliación y el tiempo en plántulas de B. alicastrum durante 4, 8, 12 y 24 meses en una selva neotropical en Calakmul, Campeche, México. Las flechas indican los porcentajes máximos y mínimos aproximados de supervivencia estimada. Los intervalos de confianza al $95 \%$ de cada tratamiento se muestran en el Cuadro 2.

Fig. 2. Logistic regression analyzed through a GLM to estimate survival probability as a function of defoliation percentage and time in $B$. alicastrum seedlings during 4, 8, 12 and 24 months in a Mexican neotropical forest in Calakmul, Campeche, Mexico. Arrows indicate the approximate maximum and minimum estimated survival percentage. Confidence intervals at $95 \%$ in each treatment are shown in Table 2 .

(7\%) y 12 hojas $(5 \%)$, respectivamente. Sólo se encontró un caso para insectos agalladores. El tipo de daño causado por patógenos removió un área foliar total de $120.78 \mathrm{~cm}^{-2}(5 \%)$, la herbivoría por insectos defoliadores un 27.28 $\mathrm{cm}^{-2}(1.2 \%)$ y con menos de $1 \%$ del área foliar los insectos raspadores y agalladores con 18.25 $\mathrm{cm}^{-2}$ y $1.11 \mathrm{~cm}^{-2}$, respectivamente.

\section{DISCUSIÓN}

Los resultados demuestran que en condiciones naturales plántulas de $B$. alicastrum pueden compensar su crecimiento en términos

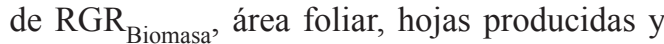
altura con una pérdida del $50 \%$ en su área foliar, e incluso la $\mathrm{RGR}_{\text {Biomasa }} \mathrm{y}$ altura con una pérdida del 90\% (McNaughton 1983, Belsky 1986).
Se explican estas diferentes respuestas compensatorias de crecimiento sobre la idea que el crecimiento compensatorio de $\mathrm{RGR}_{\text {Biomasa }}$ (crecimiento total) estaría en función del crecimiento de cada una de las estructuras vegetativas de la planta (llamado aquí mecanismo de respuestas compensatorias sinérgicas). Este potencial mecanismo de compensación sinérgica seria posible debido probablemente al papel de los carbohidratos no estructurales (CNE) almacenados en diversas estructuras de crecimiento como tallos y raíces, de mantener las funciones metabólicas estables incluso después de un evento de defoliación artificial, con lo cual a la larga esas plantas reemplazarían la pérdida de tejido fotosintético (Boege 2005, Myers y Kitajima 2007). Además, hay evidencia en condiciones naturales plántulas de $B$. alicastrum basan su crecimiento en biomasa total, sobre el obtenido en su biomasa radicular (Montgomery y Chazdon 2002).

Las respuestas de crecimiento compensatorio no han sido reportadas con base en el crecimiento total de la planta, como la $\mathrm{RGR}_{\text {Biomasa' }}$ aunque sí sobre diversas estructuras vegetativas como el área foliar (Parra-Tabla et al. 2004), producción de hojas (Canto et al. 2004), $\mathrm{RGR}_{\text {Diámetro }}$ (Kurokawa et al. 2004) y altura del tallo (Lentz y Cipollini 1998). Este estudio es el primer reporte de las respuestas de crecimiento expresado en la $\mathrm{RGR}_{\text {Biomasa }}$ después de un evento de defoliación en condiciones naturales. La $\mathrm{RGR}_{\text {Biomasa }}$ podría ser de enorme importancia para entender mejor las respuestas de crecimiento compensatorio, debido a que es una de las medidas del desempeño de las plantas más usadas (Poorter y Lambers 1991) por su sensibilidad a daños por patógenos y herbívoros (Poorter 1989).

La ausencia de crecimiento compensatorio en el área foliar y en las hojas producidas con el $90 \%$ de defoliación sugeriría la existencia potencial de un compromiso con la producción de defensas foliares. Aunque en el presente estudio no se cuantificaron las defensas químicas en hojas, diversos estudio han mostrado que la asignación de recursos para producirlas resulta detrimental para el crecimiento (Feeny 


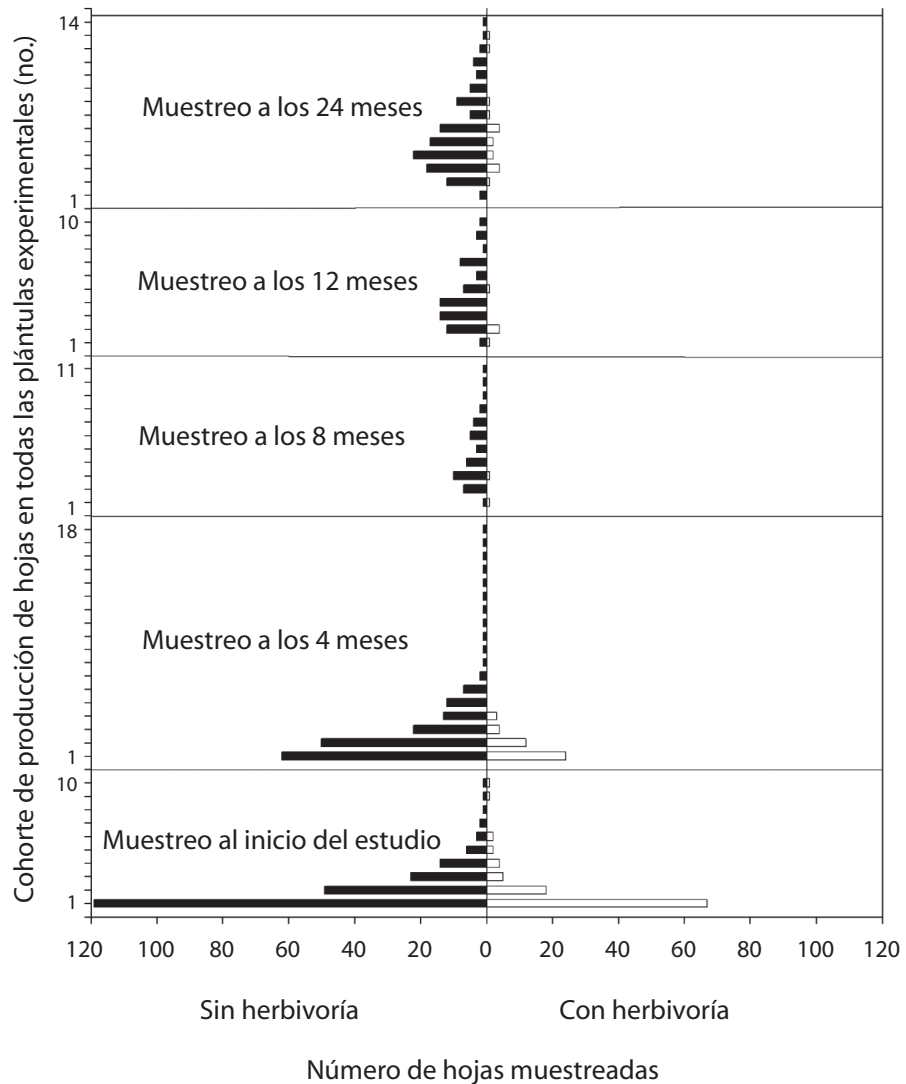

Fig. 3. Monitoreo de la herbivoría por insectos y patógenos en plántulas de B. alicastrum en una selva mediana subhúmeda durante un periodo de dos años. Se muestra la herbivoría registrada por cohorte de producción de hojas de todas las plántulas experimentales cada cuatro meses.

Fig. 3. Insect and pathogen herbivory in B. alicastrum seedlings in a tropical subhumid forest along two years of study. Herbivory records are shown by leaf cohort production of all experimental seedlings every four months.

1976, Zangerl et al. 1997). Según Coley et al. (1985) este comportamiento se agudiza para especies de sotobosque con bajas tasas de crecimiento como el caso de B. alicastrum. Ya que estas especies usan más recursos para producir defensas que para crecer. Además, un compromiso entre crecimiento versus defensas químicas resultaría probable si se observa que únicamente estructuras vegetativas como las hojas se afectaron directamente por la defoliación, y en las cuales se han encontrado defensas químicas como los fenoles foliares (H.S. Ballina-Gómez, datos no publicados). Asimismo, en la selva de Calakmul, México, las plántulas de $B$. alicastrum son consumidas naturalmente por mamíferos como el tapir (Tapirus bairdii Gill 1985, Tapiridae) y dos especies de venado (Mazama americana Rafinesque 1817, Cervidae y/o Odoicoleus virginianus Zimmermann 1780, Cervidae) (Obs. personal), siendo posible que evolutivamente haya desarrollado una serie de estrategias de 
defensas como respuesta a estos herbívoros (Strauss y Agrawal 1999).

La probabilidad de supervivencia de las plántulas de $B$. alicastrum sorprendentemente se incrementó con la defoliación. Esto podría sugerir que la defoliación aplicada pudo haber inducido una potencial producción de defensas químicas las cuales habrían disminuido la palatabilidad de las hojas y por tanto la ingesta de los herbívoros mamíferos, resultando en una mayor probabilidad de vida (Agrawal 1999). La inducción de defensas a través de la defoliación artificial es posible ya que, similar a la defoliación natural, se producen señales sistémicas resultado de las heridas (Schmelz et al. 2003). Estudios como el de Abdala-Roberts y ParraTabla (2005) muestran que en condiciones naturales la inducción de defensas es posible. En su experimento mediante defoliación artificial del 50 y $100 \%$ sobre las hojas de la especie tropical Cnidoscolus aconitifolius (Mill) I.M. Johnstone (Euphorbiaceae) observaron que esta logró producir tricomas, usados por la planta como defensas físicas contra diversos herbívoros. Asimismo, Massey et al. (2005) reportaron que la especie tropical Shorea leprosula Miq. (Dipterocarpaceae) indujo la producción de fenoles foliares totales como defensas químicas, con una defoliación artificial del 30\%.

El principal tipo de herbivoría que sufrieron las plántulas procedentes de cohortes de B. alicastrum en la selva de Calakmul fue el causado por los mamíferos. Otros estudios en especies de selvas tropicales han reportado resultados similares (Howe 1990, Osunkoya et al. 1992). La herbivoría por mamíferos fue más incidente y removió más área foliar, como característica principal de esta herbivoría fue la remoción total de la hojas quedando en pie sólo el tallo. Este tipo de herbivoría se concentró en áreas pequeñas (hasta 60 plántulas defoliadas en un $1 \mathrm{~m}^{-2}$ ) pero con grandes bancos de plántulas. Notablemente numerosas plántulas que sufrieron herbivoría por mamíferos hasta en un $100 \%$, sobrevivieron e incluso produjeron al menos una hoja en un tiempo de cuatro meses después de sufrir la herbivoría. La herbivoría por insectos y patógenos fue secundaria respecto a la causada por mamíferos. A pesar que gran número de hojas presentaron lesiones visibles de insectos y patógenos, no hubo ningún efecto aparente en la disminución del crecimiento. Los daños por patógenos fueron más frecuentes que la herbivoría por insectos. Resultados similares reportaron De la Cruz y Dirzo (1987) en una selva húmeda en Veracruz, México. Ellos encontraron que plántulas de $B$. alicastrum presentaron una mayor frecuencia de herbivoría originada por patógenos, y en menor medida por insectos.

En este estudio no se cuantificaron ni los CNE ni algún tipo de defensas químicas en las estructuras vegetativas medidas, por lo tanto, no se podría asegurar que los resultados aquí expuestos serian producto de estos factores. Aunque sí indican la presencia de un mecanismo de asignación de recursos que dirigiría las respuestas de crecimiento y supervivencia en las plántulas de $B$. alicastrum. Futuras investigaciones deberían ser enfocadas a discernir estos mecanismos, mediante la cuantificación de los CNE y la producción de defensas. En conclusión, con este estudio se mostró que $B$. alicastrum puede presentar un crecimiento compensatorio a nivel de toda la planta $\left(\mathrm{RGR}_{\text {Biomasa }}\right)$, potencialmente en función de un mecanismo sinérgico en el crecimiento de las diferentes estructuras vegetativas de la planta.

\section{AGRADECIMIENTOS}

Agradecemos al director de la Reserva de Calakmul Fernando Durand por su apoyo administrativo y logístico. Agradecemos también a Víctor Parra-Tabla, Sergio Peraza-Sánchez, K. Silveira, J. Montero, D. Pérez-Salicrup, G. Barrantes y A. Farji-Brener por sus comentarios y sugerencias. A Gerardo Polanco, Felix Dzul, Elizabeth Osorio, Manuela Tamayo, Esteban Pacheco y Celene Espadas por su ayuda en el trabajo de campo. Este estudio fue apoyado por la beca de doctorado del Consejo Nacional de Ciencia y Tecnología otorgada a H.S. Ballina-Gómez (Beca no. 183267) y por el Centro de Investigación Científica de Yucatán A.C. 


\section{RESUMEN}

Se estudiaron las respuestas de crecimiento, supervivencia y los diferentes tipos de herbivoría sobre plántulas de $B$. alicastrum en una selva neotropical de México. Se seleccionaron 122 plántulas de una población las cuales se asignaron a tres tratamientos de defoliación: control o $0 \%$ $(n=21), 50 \%(n=51)$ y $90 \%(n=50)$. Cada 4 meses durante dos años se midió su crecimiento (en términos de la tasa relativa de crecimiento en biomasa, área foliar, hojas producidas y altura) y supervivencia. Además cada 12 meses, mediante el uso de una cuadricula de $2 \mathrm{~mm}^{-2}$, se evaluó la herbivoría causada por patógenos e insectos. También dentro de una parcela de $500 \mathrm{~m}$ largo x $10 \mathrm{~m}$ de ancho se evaluó en 1095 plántulas de 3 meses de edad la herbivoría por mamíferos. En la misma parcela pero en 113 plántulas se estimó la herbivoría por patógenos e insectos. Las plántulas defoliadas al $50 \%$ compensaron su crecimiento en todas las estructuras medidas, pero sólo la tasa relativa de crecimiento y altura lo hicieron con la defoliación al $90 \%$. La tasa relativa de crecimiento y el área foliar disminuyeron gradualmente con el tiempo, contrario a la altura con un patrón opuesto. El número de hojas producidas no se afectó por el tiempo. La probabilidad estimada de supervivencia incrementó hasta un $95 \%$ con la defoliación y disminuyó hasta un $37 \%$ a los 24 meses. La herbivoría por mamíferos fue el más incidente y severo, en algunos casos hasta un $100 \%$ de defoliación. En tanto la causada por patógenos e insectos fue secundaria. El crecimiento compensatorio en área foliar, producción de hojas y altura sugieren un mecanismo compensatorio sinérgico expresado a nivel del crecimiento individual de la planta (tasa de crecimiento en biomasa). Al mismo tiempo junto con la supervivencia sugiere un posible compromiso entre las respuestas de crecimiento a nivel de la hoja - área foliar y hojas producidas - versus producción de defensas químicas.

Palabras clave: Brosimum alicastrum, crecimiento compensatorio, defoliación, herbivoría, tasa relativa de crecimiento, supervivencia de plántulas.

\section{REFERENCIAS}

Abdala, L. \& V. Parra. 2005. Artificial defoliation induces trichome production in the tropical shrub Cnidoscolus aconitifolius (Euphorbiaceae). Biotropica 37: 251257.

Agrawal, S. 1999. Induced responses to herbivory in wild radish: effects on several herbivores and plant fitness. Ecology 80: 1713-1723.

Arriaga, L., J.M. Espinoza, C. Aguilar, E. Martinez, L. Gómez \& E. Loa. 2000. Regiones terrestres prioritarias de México. CONABIO. Mexico D.F., México. $609 \mathrm{p}$.
Augspurger, C.K. 1984. Seedlings survival of tropical tree species: interactions of dispersal distance, light-gaps, and pathogens. Ecology 65: 1705-1712.

Augspurger, C.K. \& K. Kitajima. 1992. Experimental studies of seedling recruitment from contrasting seed distributions. Ecology 73: 1270-1284.

Belsky, A.J. 1986. Does herbivory benefit plants? A review of the evidence. Am. Nat. 127: 870-892.

Boege, K. 2005. Influence on plant ontogeny on compensation to leaf damage. Amer. J. Bot. 92: 1632-1640.

Canto, A., V. Parra. \& J.G. Garcia. 2004. Variations in leaf production and floral display of Anthurium schlechtendalii (Araceae) in response to herbivory and environment. Funct. Ecol. 18: 692-699.

Carabias J., E. Provencio, J. De La Maza \& J.B. Rodríguez. 2000. Programa de Manejo Reserva de la Biosfera de Calakmul. Instituto Nacional de Ecología, México D.F., México. 270 p.

Clark, D.B. \& D.A. Clark. 1989. The role of physical damage in a seedling mortality regime of a neotropical rain forest. Oikos 55: 225-230.

Coley, P.D., J.P. Bryant \& F.S. Chapin III. 1985. Resource availability and plant antiherbivore defense. Science 230: 895-899.

Coley, P.D. \& T.M. Aide. 1991. Comparison of herbivory and plant defenses in temperate and tropical broad-leaved forests, p. 25-49. In P.W. Price, T.M. Lewinsohn, G.W. Wilson \& W.W. Benson (eds.). Plant-Animal interactions: Evolution ecology in tropical and temperate regions. Wiley, New York, New York, EEUU.

Coley, P.D. \& J.A. Barone. 1996. Herbivory and plant defenses in tropical forest. Ann. Rev. Ecol. Syst. 27: 305-335.

Connell, J.H. 1971. On the role of natural enemies in preventing competitive exclusion in some marine animals and in rain forest trees, p. 298-310. In P.J. den Boer \& G.R. Grandwell (eds.). Dynamics of population. Centre for Agricultural Publications and Documentation, Wageningen, The Netherlands.

Crawley, R.J. 1989. Insect herbivores and plant population dynamics. Ann. Rev. Entomol. 34: 531-564.

De la Cruz, M. \& R. Dirzo. 1987. A survey of the standing levels of herbivory in seedlings from a Mexican Rain Forest. Biotropica 19: 98-106. 
Feeny, P. 1976. Plant apparency and chemical defense, p.1-40. In J.W. Wallace \& R.L. Mansell (eds.). Biochemical interactions between plants and insects. Plenum, New York, New York, EEUU.

García, E. 1988. Modificaciones al sistema de clasificación climática de Kôpen (para adaptarlo a las condiciones de la República Mexicana). Offset Larios, México D.F., México. 165 p.

Hjalten, J., K. Danell \& L. Ericson. 1994. The impact of herbivory and competition on the phenolic concentration and palatability of juvenile birches. Oikos 71: 416-422.

Howe, H.F. 1990. Survival and growth of juvenile Virola surinamensis in Panama: effects of herbivory and canopy closure. J. Trop. Ecol. 6: 259-280.

Howe, H. F. \& J. Smallwood. 1982. Ecology of seed dispersal. Ann. Rev. Ecol. Syst. 13: 201-28.

Hunt, R. 1978. Basic growth analysis: Plant growth analysis for beginners. Unwin Hyman, London, UK. 112 p.

Huntly, N. 1991. Herbivores and the dynamics of communities and ecosystems. Ann. Rev. Ecol. Syst. 22: 477-503.

Janzen, D.H. 1970. Herbivores and the number of tree species in tropical forests. Am. Nat. 104: 501-528.

Kurokawa, H., Y. Kitahashi, T. Koike, J. Lai \& T. Nakashizuka. 2004. Allocation to defense or growth in dipterocarp forest seedlings in Borneo. Oecologia 140: $261-270$

Lentz, K.A. \& D.F. Cipollini Jr. 1998. Effect of light and simulated herbivory on growth of endangered northeastern bulrush, Scirpus ancistrochaetus Schuyler. Plant Ecol. 139: 125-131.

Marquis, R. J. 1984. Leaf herbivores decrease fitness of a tropical plant. Science 226: 537-539.

Martínez, E. \& C. Galindo. 2002. La vegetación de Calakmul, Campeche, México: clasificación, descripción y distribución. Bol. Soc. Bot. México 71: 7-32.

Martínez, E., S.M. Souza \& C.H. Ramos. 2001. Listados Florísticos de México. XXII. Región de Calakmul Campeche. Instituto de Biología, UNAM. México D.F., México. 55 p.

Massey, F.P., M.C. Press \& S.E. Hartley. 2005. Long and short term induction of defences in seedlings of
Shorea leprosula (Dipterocarpaceae): support for the carbon:nutrient balance hypothesis. J. Trop. Ecol. 21: 195-201.

McCullagh, P. \& J.A. Nelder. 1989. Generalized linear model. Chapman \& Hall, London, U.K. 511 p.

McNaughton, S.J. 1983. Compensatory growth as a response to herbivory. Oikos 40: 329-336.

Montgomery, R. \& R. Chazdon. 2002. Light gradient partitioning by tropical tree seedlings in the absence of canopy gaps. Oecologia 131: 165-174.

Morales, R. J. \& R.S. Magaña. 2001. Fuentes de impacto, necesidades de investigación científica y monitoreo en Calakmul, Campeche. PRONATURA Península de Yucatán y The Nature Conservancy. México D.F., México. 81 p.

Myers, J.A. \& K. Kitajima. 2007. Carbohydrate storage enhances seedling shade and stress tolerance in a neotropical forest. J. Ecol. 95: 383-395.

Orellana, R., G. Islebe \& C. Espadas. 2003. Presente, pasado y futuro de los climas de la Península de Yucatán, p. 37-52. In P. Colunga \& A. Larqué (eds.). Naturaleza y sociedad en el área Maya. Pasado, presente y futuro. Academia Mexicana de Ciencias y CICY, Mérida, Yucatán, México.

Osunkoya, O.O., J.E. Ash, M.E. Hopkins \& A.W. Graham. 1992. Factors affecting survival of tree seedlings in North Queensland rainforest. Oecologia 91: 569-578.

Parra, V., V. Rico \& M. Carvajal. 2004. Effect of defoliation on leaf growth, sexual expression and reproductive success of Cnidoscolus aconitifolius (Euphorbiaceae). Plant Ecol. 173: 153-160.

Poorter, H. 2002. Plant growth and carbon economy, p. 1-6. In Encyclopedia of life sciences. Nature, London, UK.

Poorter, H. 1989. Interspecific variation in relative growth rate: on ecological causes and physiological consequences, p. 45-68. In H. Lambers, M.L. Cambridge, H. Konings \& T.L. Pons (eds.). Causes and consequences of variation in growth rate and productivity of higher plants. SPB Academic Publishing, The Hague, The Netherlands.

Poorter, H. \& H. Lambers. 1991. Is interspecific the variation in relative growth rate positively correlated with biomass allocation to the leaves? Am. Nat. 138: 1264-1268. 
Schmelz, E.A., Alborn, H.T. \& J.H. Tumlinson. 2003. Synergistic interactions between volicitin, jasmonic acid and ethylene mediate insect-induced volatile emission in Zea mays. Physiol. Plant 117: 403-412.

SPSS. 2007. SPSS Version 16. SPSS, Chicago, IL, EEUU.

Statistica. 2004. Statistica Version 7. Statsoft, Tulsa, OK, EEUU.

Stone, C. \& P.E. Bacon. 1995. Influence of insect herbivory on the decline of black box (Eucalyptus largiflorens). Australian J. Bot. 43: 555-564.
Strauss, S. \& A. Agrawal. 1999. The ecology and evolution of plant tolerance to herbivory. TREES. 14: 179-185.

Wendt, T. 1993. Composition, floristic affinities, and origins of the canopy tree flora of the Mexican Atlantic slope rain forest, p. 595-680. In T.P.R. Ramamoorthy, R. Bye, A. Lot \& J. Fa (eds.). Biological diversity of Mexico: origins and distribution. Oxford University, New York, New York, EEUU.

Zangerl, A.R., A.M. Arntz \& M.R. Berenbaum. 1997. Physiological price of an induced chemical defense: photosynthesis, respiration, biosynthesis, and growth. Oecologia 109: 433-441. 
